\title{
Recoartación de la aorta en pacientes sometidos a angioplastia percutánea con o sin implantación de stent
}

\author{
Recoarctation of the aorta in patients subjected to ballon angioplasty with or without \\ implementation of stent
}

\author{
María A. Cañas-Galvis ${ }^{1}$, Rafael Lince-Varela ${ }^{2 *}$, Luis H. Díaz-Medina², Rafael Correa ${ }^{2}$ y Diana Restrepo \\ ${ }^{1}$ Facultad de Medicina, Universidad CES; ${ }^{2}$ Servicio de Cardiología Pediátrica, Clínica CardioVID. Medellín, Colombia
}

\begin{abstract}
Resumen
Objetivo: La coartación de la aorta puede ser tratada quirúrgicamente o con angioplastia con balón. El objetivo de nuestro estudio fue describir los resultados postangioplastia percutánea con balón con o sin implantación de stent para coartación de la aorta y establecer la incidencia de recoartación en el seguimiento. Método: Estudio de cohorte, se incluyeron pacientes de cualquier edad en un periodo de seguimiento de nueve años. Resultados: Se incluyeron 89 pacientes, un 69.0\% hombres. La media de seguimiento para todos los participantes fue de 33.66 meses. Al $32.5 \%$ se les implantó un stent en angioplastia; de los cuales el $24.1 \%$ se recoartaron durante el seguimiento. En el grupo sin stent se recoartaron un $36.6 \%$. No hubo diferencia significativa en las curvas de supervivencia de los dos grupos $(p=0.889)$. Conclusiones: La implantación de stent durante la angioplastia con balón para tratar coartación de aorta no influyó en la incidencia de recoartación de la aorta; pero factores como la hipertensión arterial preangioplastia y el gradiente final de angioplastia $>20 \mathrm{mmHg}$ se asoció con recoartación de la aorta.
\end{abstract}

Palabras clave: Angioplastia coronaria con balón. Coartación aórtica. Cardiopatías congénitas. Stents. Pediatría. Estudios de seguimiento.

\begin{abstract}
Objective: Coarctation of the aorta can be treated surgically or with balloon angioplasty. The objective of our study was to describe the results after percutaneous balloon angioplasty with or without stent implantation for coarctation of the aorta and establish the incidence of recovery during follow-up. Method: Cohort study. 89 patients of any age where included in a follow up period of nine years. Results: Of the 89 patients included in the study, $69.0 \%$ were male. The mean follow-up for all participants was 33.66 months. $32.5 \%$ of the patients had a stent implanted during the angioplasty procedure; of which $24.1 \%$ suffered recoarctation during follow-up. In the group without stent implantation, $36.6 \%$ suffered recoarctation. There was not significant difference in the survival curves of the two groups $(p=0.899)$. Conclusions: Stent implantation during balloon angioplasty to treat aortic coarctation did not influence in the incidence of aortic recoarctation; but factors such as preangioplasty arterial hypertension and the final angioplasty gradient $>20 \mathrm{mmHg}$ is associated with aortic recoarctation.
\end{abstract}

Key words: Angioplasty. Balloon. Coronary. Coarctation. Aortic. Heart defects. Congenital. Stents. Pediatrics. Follow-Up studies.

Correspondencia:

${ }^{*}$ Rafael Lince-Varela

E-mail: rafalince@yahoo.com
Disponible en internet: 29-10-2021 Arch Cardiol Mex. 2021;91(4):444-452 www.archivoscardiologia.com

(c) 2020 Instituto Nacional de Cardiología Ignacio Chávez. Publicado por Permanyer. Este es un artículo open access bajo la licencia CC BY-NC-ND (http://creativecommons.org/licenses/by-nc-nd/4.0/). 


\section{Introducción}

La coartación de aorta se define como una estrechez congénita de la aorta torácica proximal ${ }^{1}$, la cual explica entre el 8 y el $10 \%$ de todos los defectos cardiacos congénitos $^{2}$. Este defecto permite el aumento de la poscarga del ventrículo izquierdo con un aumento de la presión arterial en región proximal y disminución de la perfusión en la parte inferior del cuerpo ${ }^{3}$. De no tratarse, la coartación de aorta se asocia con importante morbilidad y disminución de la sobrevida, por complicaciones asociadas a hipertensión arterial, insuficiencia cardiaca congestiva, infarto agudo de miocardio, ataque cerebrovascular, infarto de miocardio, endocarditis infecciona y ruptura aórtica².

El primer tratamiento instaurado fue la resección quirúrgica con anastomosis terminoterminal, descrita por primera vez por Crafoord en $1945^{3}$. Posteriormente, estudios experimentales de Sos, et al. en 1979 demostraron la posibilidad de dilatar la coartación aórtica en especímenes humanos post mortem ${ }^{4}$, y desde 1982 este procedimiento ha ganado reconocimiento como una intervención segura, menos invasiva ${ }^{5} \mathrm{y}$ efectiva en el $79 \%$ de pacientes con recoartación de aorta, con una mortalidad entre el 0.7 y el $2.5 \%$.

En un inicio, la angioplastia con implantación de stent fue propuesta como tratamiento para la coartación recurrente de aorta ${ }^{7,8}$, sin embargo, algunos la han propuesto para el tratamiento de las coartaciones nativas $^{9,10}$.

La angioplastia con balón en los menores de 1 año de edad ha mostrado una alta incidencia de reestenosis y el desarrollo de aneurismas ${ }^{11}$, por lo que en la década de 1990 se introdujo la terapia intravascular de implantación de stents metálicos desnudos, que, al proporcionar una prótesis endovascular rígida, mantuvieran un diámetro adecuado del vaso sanguíneo en el segmento de la coartación, lo cual ha mostrado buenos resultados ${ }^{5}$. Por tanto, la implantación de stent fue propuesta inicialmente para la coartación recurrente de aorta ${ }^{7-8}$ sin embargo algunos la han propuesto para el tratamiento de las coartaciones nativas ${ }^{6-10}$.

En Medellín (Colombia) las primeras angioplastias con balón se realizaron a mediados de la década de 1990 y la primera implantación de stent a finales de dicha década, sin que se disponga de información de los resultados clínicos. El objetivo de nuestro estudio fue comparar la reestenosis de la aorta en dos grupos de pacientes sometidos a angioplastia con 0 sin implantación de stent.

\section{Material y métodos}

\section{Tipo de estudio}

Se realizó un estudio de cohorte. Todos los pacientes fueron sometidos a angioplastia con balón con o sin implantación de stent para tratamiento de coartación de aorta, y se les realizó seguimiento por un periodo de tiempo máximo de 9 años y mínimo de 20 meses para determinar los casos de recoartación de la aorta.

\section{Contexto}

El estudio incluyó pacientes de la Clínica CardioVID, en la ciudad de Medellín (Colombia), a quienes se les realizó un primer procedimiento de angioplastia percutánea con balón y/o stent en el periodo comprendido entre enero de 2009 y diciembre de 2017. Para el seguimiento de los pacientes incluidos en el 2017, el seguimiento se extendió hasta agosto de 2019. Siete de los pacientes intervenidos no regresaron a la institución para seguimiento, pero fueron contactados telefónicamente y ninguno requirió nuevas intervenciones.

\section{Participantes}

Se incluyeron la totalidad de los pacientes de ambos sexos, sin límite de edad, con diagnóstico de coartación de la aorta que durante el periodo del estudio habían sido atendidos en la Clínica CardioVID, por el grupo de cardiología pediátrica, los cuales fueron identificados a partir de la base de datos elaborada por los hemodinamistas investigadores, la cual fue completada inmediatamente después de realizar cada cateterismo.

En cuanto a los grupos de comparación, se conformaron dos grupos: uno con angioplastia más implantación de stent y otro con angioplastia sin implantación de stent. El desenlace primario fue la recoartación de la aorta.

\section{Variables}

La variable dependiente para el estudio fue recoartación de la aorta. Otras variables incluidas fueron: demográficas (edad, sexo), nutricionales (peso, talla), clínicas (diagnósticos de cardiopatías asociadas, hipertensión arterial, uso de antihipertensivos), ecográficas (gradiente preangioplastia y postangioplastia), relacionadas con el procedimiento (gradiente preangioplastia y postangioplastia, tipo de stent, complicaciones por el procedimiento) y de seguimiento (tiempo en meses a partir de la angioplastia con 
balón hasta la recoartación). En el análisis estadístico, los factores considerados como posibles confusores fueron la edad menor de 6 meses, implantación de stent, sexo masculino y coartación nativa.

Respecto al éxito y desenlace primario, el éxito en la angioplastia se definió como un gradiente residual $\leq 20 \mathrm{mmHg}$

\section{Definiciones}

Para este estudio la coartación de aorta se definió como un gradiente sistólico en reposo $\geq 20 \mathrm{mmHg}$ en hemodinámica. Si posterior a reparación por angioplastia con balón, con o sin implantación de stent, la aorta vuelve a presentar estenosis con gradiente $\geq$ de $20 \mathrm{mmHg}$ y síntomas clínicos ${ }^{12}$, nos referimos a recoartación. Además, la muerte relacionada con angioplastia se definió como toda muerte ocurrida en las siguientes 24 horas luego de la angioplastia con balón con o sin implantación de stent para corregir la coartación nativa o recoartación de aorta.

\section{Fuentes de datos y medidas}

La presión arterial fue tomada con esfigmomanómetro. Los gradientes de presión fueron obtenidos a partir de un ecocardiógrafo Philips Epic y Vivid 7.

\section{Procedimientos}

\section{HeModinámicos}

La angioplastia con balón y/o implantación de stent fue realizada bajo anestesia general. En el tiempo del estudio no se presentaron diferencias importantes en las técnicas o en el equipo empleado y los hemodinamistas fueron los mismos en el tiempo del seguimiento. Se administró heparina $100 \mathrm{Ul} / \mathrm{kg}$ de peso, por vía intravenosa tan pronto como se introdujo la guía arterial. La angiografía del arco aórtico permitió medir los diámetros de la aorta en varios sitios que incluyeron aorta transversa, istmo aórtico, zona de la coartación y la aorta a nivel del diafragma. El catéter balón y/o stent fue avanzado hasta el arco aórtico a la zona de la coartación, donde se infló a las atmósferas sugeridas por el fabricante del dispositivo (generalmente menor a 6 atmósferas). Se infla con contraste diluido hasta que desparece la cintura que crea la coartación en el balón, se espera alrededor de 10 segundos, se desinfla y se retira el balón, lo cual se repite tres veces para optimizar el resultado final.
La implantación del stent es similar. Luego de la toma de las medidas se lleva el stent montado en un balón, se infla el balón y se libera el stent en el sitio de la coartación. Luego de retirar el balón, se pasa un catéter sobre la guía para medir la presión aórtica y realizar el angiograma. El procedimiento se repite usando un diámetro de balón un milímetro mayor cuando el resultado no es satisfactorio. El tamaño del balón no excede el diámetro aórtico medido a nivel del diafragma inicial y no más de 2 milímetros.

\section{ECOCARDIOGRÁFICOS}

El seguimiento de los pacientes se realizó mediante ecocardiografía transtorácica, realizada con dos equipos (Philips Epic y Vivid 7). Se definió recoartación como la presencia de un gradiente máximo $>36 \mathrm{mmHg}$ o gradiente medio $>20 \mathrm{mmHg}$ y/o presencia de flujo diastólico reverso a nivel de la aorta descendente (se necesita referencia mínima 1, ideal 2).

\section{Sesgos}

El sesgo de selección se controló al incluir todos los participantes de forma consecutiva que fueron sometidos a angioplastia con balón en el periodo de estudio. El sesgo de información se minimizó por la alta calidad del dato al provenir directamente de quien realizó el procedimiento $(\mathrm{RL})$. Además, cada historia clínica fue revisada retrospectivamente para verificar la información. En los casos en los que se tenía duda, el investigador principal verificó con quien extrajo la información cualquier inconsistencia de esta. Respecto al sesgo de seguimiento, se llamó a los que se desaparecieron en el seguimiento y se verificó el motivo de no volver al seguimiento, y si habían sido sometidos a nuevos procedimientos de angioplastia o cirugía por recoartación de la aorta. También se hizo imputación múltiple de los datos perdidos y se compararon los resultados. Finalmente, la base de datos fue depurada por dos de los investigadores (AC, DR), uno de ellos con experiencia en el manejo de bases de datos, buscando registros duplicados o inconsistentes. Además, para el sesgo de confusión se utilizó el método multivariante de regresión de Cox.

\section{Tamaño muestral}

Todos los casos que de forma consecutiva ingresaron a la institución con el diagnóstico de coartación de aorta y fueron sometidos a angiografía percutánea con 
balón durante el periodo de estudio fueron incluidos en el estudio.

\section{Variables cuantitativas}

Las variables cuantitativas en el análisis fueron tratadas de la siguiente forma, según el criterio clínico del hemodinamista $(\mathrm{RL})$ en función del mayor riesgo para la recoartación de la aorta:

- Edad. Se tomó de forma continua y luego se transformó en categorías de edad (neonatos, lactantes, preescolares, escolares, adolescentes y adultos), también se recategorizó en mayores y menores de 6 meses.

- Gradiente preangioplastia y postangioplastia. Se tomó el dato registrado en la angioplastia en $\mathrm{mmHg}$. Posteriormente se tomó como valor de referencia 20 $\mathrm{mmHg}$ y a partir de este valor se recategorizó como normal o con estenosis.

- Tiempo de seguimiento luego de la angioplastia. Se tomó en meses a partir de la angioplastia índice y se midió hasta la finalización del periodo de observación del estudio o hasta el evento de interés (recoartación de la aorta).

\section{Métodos estadísticos}

Se realizó un análisis descriptivo de las variables sociodemográficas y clínicas. Como indicadores epidemiológicos se empleó la incidencia cruda de recoartación de la aorta; también se calculó la densidad de incidencia. Las variables cualitativas se presentaron con frecuencias absolutas y relativas. Para las variables cuantitativas se emplearon medidas de resumen como mediana y rango intercuartílico $(R \mid Q)$, porque las variables cuantitativas no tuvieron distribución normal según el test de Shapiro-Wilks. Se buscó posible asociación entre la recoartación de la aorta y las variables incluidas en el estudio (edad menor de 6 meses, gradiente final angiográfico, implantación de stent, coartación nativa). Con el método de Kaplan-Meier se estimó la función del tiempo transcurrido hasta la aparición de la recoartación. Las curvas de supervivencia fueron comparadas mediante Log-rank test.

Se realizó el análisis multivariante usando el modelo de riesgo proporcional de Cox (método introducir) para estimar las hazard ratios ajustadas de presentar recoartación de aorta con aquellas variables que habían mostrado $p<0.20$ en el modelo bivariante. Se consideraron significativos valores de $p<0.05$ y se presentan las hazard ratio con intervalo de confianza

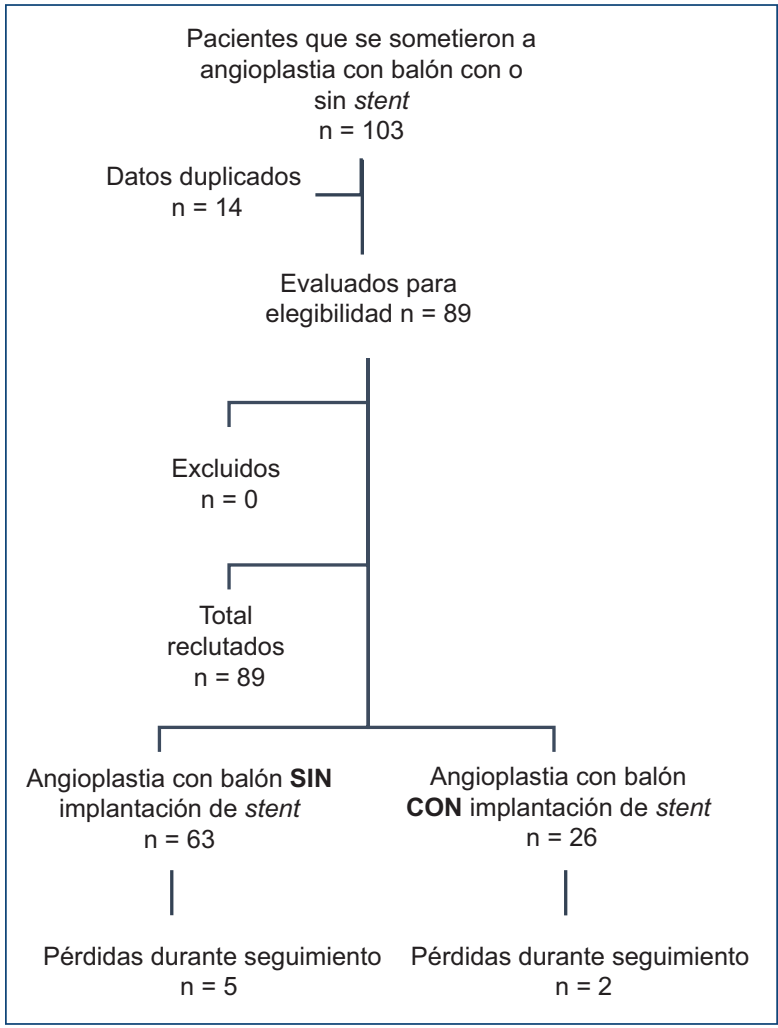

Figura 1. Diagrama de flujo de los pacientes ingresados en los grupos de estudio.

del 95\% (IC 95\%). Para el análisis de la información se utilizó el software SPSS ${ }^{\circledR}$ versión 21.0 (SPSS Inc; Chicago, Illinois, EE.UU.), licencia amparada por la Universidad CES.

\section{Aspectos éticos}

Investigación sin riesgo según la normativa colombiana. Estudio aprobado por el Comité de Investigación de la Universidad CES y de la Clínica CardioVID.

\section{Resultados}

\section{Participantes}

La muestra quedó conformada por 103 pacientes. En 14 casos se encontró duplicación de los datos. Se realizaron 89 angioplastias primarias, que constituyen la totalidad de la muestra. De estos pacientes, 7 no volvieron a la institución; se logró contactar telefónicamente con el $100 \%$ de ellos y se verificó que ninguno se había recoartado para el momento del contacto (octubre de 2019) (Fig. 1). 
Tabla 1. Características demográficas de los participantes

\begin{tabular}{|c|c|c|c|}
\hline Variable & $\begin{array}{c}\text { Angioplastia CON } \\
\text { implantación de stent n (\%) }\end{array}$ & $\begin{array}{c}\text { Angioplastia SIN } \\
\text { implantación de stent } n(\%)\end{array}$ & Valor $p$ \\
\hline $\begin{array}{l}\text { Edad en meses } \\
\text { (Me, RIQ) } \\
\text { Mínimo } \\
\text { Máximo }\end{array}$ & $\begin{array}{c}156.0(60) \\
2.0 \\
468.0\end{array}$ & $\begin{array}{c}36.00(56.0) \\
0.3 \\
204.0\end{array}$ & $<0.001$ \\
\hline $\begin{array}{l}\text { Sexo } \\
\text { Hombre } \\
\text { Mujer }\end{array}$ & $\begin{array}{l}20(69.0) \\
9(31.0)\end{array}$ & $\begin{array}{l}40(66.7) \\
20(33.3)\end{array}$ & 0.219 \\
\hline $\begin{array}{l}\text { Grupos de edad } \\
\text { Neonato } \\
\text { Lactante } \\
\text { Preescolar } \\
\text { Escolar } \\
\text { Adolescente y mayor }\end{array}$ & $\begin{array}{c}0(0.0) \\
1(3.4) \\
0(0.0) \\
11(37.9) \\
17(58.6)\end{array}$ & $\begin{array}{c}6(10.0) \\
24(40.0) \\
19(31.7) \\
8(13.3) \\
3(5.0)\end{array}$ & 0.389 \\
\hline $\begin{array}{l}\text { Edad } \\
\text { Menor de } 6 \text { meses } \\
\text { Mayor de } 6 \text { meses }\end{array}$ & $\begin{array}{c}1(3.4) \\
28(96.6)\end{array}$ & $\begin{array}{l}19(31.7) \\
41(68.3)\end{array}$ & 0.518 \\
\hline $\begin{array}{l}\text { Peso kilogramos } \\
\text { (Me, RIQ) } \\
\text { Mínimo } \\
\text { Máximo }\end{array}$ & $\begin{array}{c}48.0(25.0) \\
1.6 \\
92.0\end{array}$ & $\begin{array}{c}12.3(25.0) \\
2.6 \\
77.0\end{array}$ & $<0.001$ \\
\hline $\begin{array}{l}\text { Coartación nativa } \\
\text { Sí } \\
\text { No }\end{array}$ & $\begin{array}{l}16(55.2) \\
13(44.8)\end{array}$ & $\begin{array}{l}29(48.3) \\
31(51.7)\end{array}$ & 0.387 \\
\hline $\begin{array}{l}\text { Aorta bivalva } \\
\text { Sí } \\
\text { No }\end{array}$ & $\begin{array}{l}19(65.5) \\
10(34.5)\end{array}$ & $\begin{array}{l}45(75.0) \\
15(25.0)\end{array}$ & 0.087 \\
\hline $\begin{array}{l}\text { Cardiopatías asociadas } \\
\text { Simples } \\
\text { Complejas } \\
\text { No tiene otra cardiopatía }\end{array}$ & $\begin{array}{c}19(65.5) \\
0(0.0) \\
10(34.5)\end{array}$ & $\begin{array}{c}9(15.0) \\
45(75.0) \\
6(10.0)\end{array}$ & 0.202 \\
\hline $\begin{array}{l}\text { Hipertensión arterial } \\
\text { Sí } \\
\text { No }\end{array}$ & $\begin{array}{c}25(86.2) \\
4(13.8)\end{array}$ & $\begin{array}{l}37(61.7) \\
23(38.3)\end{array}$ & 0.143 \\
\hline $\begin{array}{l}\text { Antihipertensivos preangioplastia } \\
\text { Sí } \\
\text { No }\end{array}$ & $\begin{array}{l}19(65.5) \\
10(34.5)\end{array}$ & $\begin{array}{l}32(53.3) \\
28(46.7)\end{array}$ & 0.276 \\
\hline $\begin{array}{l}\text { Antihipertensivos postangioplastia } \\
\text { Sí } \\
\text { No }\end{array}$ & $\begin{array}{l}21(72.4) \\
8(27.6)\end{array}$ & $\begin{array}{l}37(61.7) \\
23(38.3)\end{array}$ & 0.319 \\
\hline
\end{tabular}

Me: Mediana; RIQ: Rango intercuartílico.

\section{Datos descriptivos}

Se incluyeron 89 pacientes. En el grupo de implantación de stent 29 pacientes, de los cuales el $69.0 \%$ eran hombres, y en el grupo sin stent 60 pacientes, de los cuales el $66.7 \%$ fueron hombres. Solo seis neonatos fueron intervenidos y todos en el grupo sin stent. Se implantó solo un stent en un lactante menor frente a 24 lactantes que tuvieron angioplastia sin stent. En las tablas 1 y 2 se muestran las características sociodemográficas de los participantes en el estudio.

\section{Periodo de seguimiento}

Durante el seguimiento desde enero de 2009 hasta diciembre de 2017 se siguieron 89 personas (hacer la sumatoria del tiempo total de seguimiento de las 89 personas) personas-año, con un seguimiento mínimo 
Tabla 2. Características hemodinámicas de los participantes

\begin{tabular}{|c|c|c|c|}
\hline Variable & Con stent (\%) & Sin stent (\%) & Valor p \\
\hline $\begin{array}{l}\text { Redilatación del stent } \\
\text { - Sí } \\
\text { - No }\end{array}$ & $\begin{array}{c}4(13.8) \\
25(86.2)\end{array}$ & $\begin{array}{c}2(3.3) \\
58(96.7)\end{array}$ & 0.667 \\
\hline $\begin{array}{l}\text { Tipo de cateterismo } \\
\text { - Diagnóstico } \\
\text { - Redilatación aorta } \\
\text { - Redilatación stent } \\
\text { - Implantación stent }\end{array}$ & $\begin{array}{c}1(3.4) \\
2(6.9) \\
6(20.7) \\
20(69.0)\end{array}$ & $\begin{array}{c}29(48.3) \\
27(45.0) \\
3(5.0) \\
1(1.7)\end{array}$ & 0.717 \\
\hline $\begin{array}{l}\text { Tipo de stent } \\
\text { - CP } \\
\text { - Palmaz PG } \\
\text { - Advanta V12 } \\
\text { - Integrity } \\
\text { - No se implantó stent }\end{array}$ & $\begin{aligned} 14 & (48.3) \\
2 & (6.9) \\
12 & (41.4) \\
1 & (3.4) \\
0 & (0.0)\end{aligned}$ & $\begin{array}{c}0(0.0) \\
3(5.0) \\
0(0.0) \\
0(0.0) \\
57(97.0)\end{array}$ & 0.259 \\
\hline $\begin{array}{l}\text { Acceso vascular } \\
\text { - Art. femoral } \\
\text { - Art. disección carotídea } \\
\text { - Híbrido }\end{array}$ & $\begin{aligned} 28 & (96.6) \\
1 & (3.4) \\
0 & (0.0)\end{aligned}$ & $\begin{array}{l}53(88.3) \\
6(10.0) \\
1(1.7)\end{array}$ & 0.428 \\
\hline $\begin{array}{l}\text { Implantación del stent } \\
\text { - Sí } \\
\text { - No }\end{array}$ & $\begin{array}{c}7(24.1) \\
22(75.9)\end{array}$ & $\begin{array}{l}19(31.7) \\
41(68.3)\end{array}$ & 0.464 \\
\hline $\begin{array}{l}\text { Complicaciones asociadas al procedimiento } \\
\text { - Sí } \\
\text { - No }\end{array}$ & $\begin{array}{c}3(10.3) \\
28(96.6)\end{array}$ & $\begin{array}{c}12(19.8) \\
4(83.3)\end{array}$ & 0.206 \\
\hline $\begin{array}{l}\text { Tipo de complicaciones } \\
\text { - Hematoma inguinal } \\
\text { - Disección aórtica } \\
\text { - Aneurisma aórtico } \\
\text { - Bradicardia grave } \\
\text { - Muerte } \\
\text { - Otras complicaciones } \\
\text { - No aplica }\end{array}$ & $\begin{array}{c}1(3.4) \\
0(0.0) \\
2(6.9) \\
0(0.0) \\
0(0.0) \\
0(0.0) \\
28(96.6)\end{array}$ & $\begin{array}{c}3(5.0) \\
1(1.6) \\
3(5.0) \\
1(1.6) \\
1(1.6) \\
3(5.0) \\
48(80.2)\end{array}$ & 0.693 \\
\hline Gradiente inicial angioplastia mmHg (Me. RIO) & $24.0(19.0)$ & $28.5(16.2)$ & 0.099 \\
\hline Gradiente final angioplastia $\mathrm{mmHg}$ (Me. RIQ) & $4.0(7.0)$ & $13.0(8.2)$ & $<0.001$ \\
\hline $\begin{array}{l}\text { Tiempo de seguimiento hasta recoartación } \\
\text { - Mínimo } \\
\text { - Máximo }\end{array}$ & $\begin{array}{c}24(27) \\
4.0 \\
96.0\end{array}$ & $\begin{array}{c}22.5(37.7) \\
1.0 \\
109.0\end{array}$ & 0.460 \\
\hline
\end{tabular}

Me: Mediana; RIQ: Rango intercuartílico.

de 1 mes y máximo de 109 meses, con una media para todos los participantes de 33.66 meses (desviación estándar: 30.70 ) y mediana 25.0 (RIQ: 46.5).

\section{Datos de variable de resultado}

\section{RECOARTACIÓN}

Durante el periodo de estudio al $32.5 \%$ (29) de los pacientes se les implantó un stent durante el procedimiento de angioplastia; 7 de estos pacientes (24.1\%) se recoartaron durante el seguimiento. De los 60 pacientes a los que no se les implantó stent, se recoartaron 22 (36.6\%).

Riesgo relativo (RR): a partir del riesgo de recoartación en expuestos al stent (0.24) y no expuestos al stent (0.36), se calcula el RR $=0.24$ / $0.36=0.66$.

\section{Incidencia acumulada de recoartación}

Se estimó que en un tiempo de seguimiento de 40.43 años se presentaron 26 recoartaciones de la aorta, lo 
Tabla 3. Incidencia acumulada de recoartación de la aorta en pacientes con y sin implantación de stent, según grupos de edad

\begin{tabular}{|c|c|c|c|c|c|}
\hline \multicolumn{3}{|c|}{ Grupos de edad en meses } & \multicolumn{2}{|c|}{ Recoartación } & \multirow[t]{2}{*}{ Incidencia } \\
\hline & & & Sí & No & \\
\hline \multirow{2}{*}{$\begin{array}{l}0-1 \\
\text { Neonatos }\end{array}$} & \multirow[t]{2}{*}{ Implantación del stent } & Sí & 0 & 0 & NC \\
\hline & & No & 3 & 3 & 0.5 \\
\hline \multirow{2}{*}{$\begin{array}{l}>1-24 \\
\text { Lactantes }\end{array}$} & \multirow[t]{2}{*}{ Implantación del stent } & Sí & 0 & 1 & 0 \\
\hline & & No & 6 & 18 & 0 \\
\hline \multirow{2}{*}{$\begin{array}{l}25-60 \\
\text { Pre escolar }\end{array}$} & \multirow[t]{2}{*}{ Implantación del stent } & Sí & 0 & 0 & NC \\
\hline & & No & 8 & 11 & 0.44 \\
\hline \multirow{2}{*}{$\begin{array}{l}61-144 \\
\text { Escolar }\end{array}$} & \multirow[t]{2}{*}{ Implantación del stent } & Sí & 4 & 7 & 0.36 \\
\hline & & No & 2 & 6 & 0.25 \\
\hline \multirow{2}{*}{$\begin{array}{l}>145 \\
\text { Adolescentes/adultos }\end{array}$} & \multirow[t]{2}{*}{ Implantación del stent } & Sí & 3 & 13 & 0.18 \\
\hline & & No & 0 & 4 & 0 \\
\hline
\end{tabular}

NC: No calculable.

que determina una incidencia acumulada de 64.3 nuevos eventos x 100 pacientes/año. En los pacientes con implantación de stent, se presentaron 9 eventos durante 18.16 años de seguimiento, para una incidencia acumulada de 38.54 nuevos eventos x 100 personas/año. En los pacientes sometidos a angioplastias sin implantación de stent, se presentaron 19 eventos durante un seguimiento de 22.2 años, para una incidencia acumulada de 85.35 nuevos eventos x 100 personas/año.

\section{Incidencia de recoartación de la aorta por grupos de edad}

Se realiza un análisis estratificado por grupos de edad, con su respectiva incidencia de recoartación, lo que permite identificar en cuál grupo se encuentra la mayor incidencia: en el grupo de neonatos, con un $50 \%$, seguido por el grupo de preescolar, con un $44 \%$ (Tabla 3).

\section{Discusión}

La coartación de aorta es una patología relativamente frecuente, que cuando no es corregida se asocia a disminución de la sobrevida ${ }^{3}$. A pesar de que existe controversia para elegir el tratamiento, en los últimos años los cateterismos cardiacos se han convertido en procedimientos cada vez más seguros ${ }^{13}$, dados los avances técnicos y las mejoras en la atención posterior al procedimiento en unidades de cuidados intensivos ${ }^{14}$.

En este estudio se analizaron 89 pacientes que requirieron angioplastia con balón con o sin implantación de stent en un periodo de nueve años. El hallazgo más importante de este estudio lo constituye el hecho de que si bien se presentó una menor incidencia de recoartación en el grupo con implantación de stent, y este grupo tuvo un tiempo mayor libre de recoartación, la implantación de stent durante la angioplastia con balón para tratar la coartación de la aorta no influyó significativamente en la recoartación de aorta durante el tiempo de seguimiento total. Esto difiere de otros estudios que han encontrado una menor recoartación de la aorta luego de angioplastia con balón más implantación de stent ${ }^{15,16}$. No obstante, se debe tener en consideración que la decisión de colocar o no stent para tratar la coartación de aorta se basa en múltiples variables, como el gradiente inicial, la experiencia del centro de salud, la presencia de antecedentes como hipertensión arterial, accidente cerebrovascular, estenosis grave del itsmo y la repercusión sobre el ventrículo izquierdo en el electrocardiograma o ecocardiograma, entre otros ${ }^{17}$.

Existen numerosas publicaciones que revisan las ventajas de una técnica sobre la otra, como la de Bulbul, et al. ${ }^{18}$, donde se sobresale que al implantar stent se reducen las posibilidades de embolización y el manejo de guías a través de la zona dilatada. La 
implantación de stent está catalogada erróneamente como «más agresiva» comparándola con angioplastia solo con balón; sin embargo, algunos estudios sugieren que en algunas circunstancias es preferible el uso de stent, como en el postoperatorio temprano ${ }^{19,20}$.

Algunos estudios han mostrado asociación entre la hipertensión arterial posterior al procedimiento y la recoartación de la aorta, lo cual es descrito como una complicación ${ }^{21}$ relacionada con cambios en la vasculatura del arco aórtico ${ }^{22}$. Dijkema, et al. analizaron el resultado a largo plazo de pacientes con edades entre los 3 meses y los 16 años sometidos a angioplastia con balón o cirugía para coartación de la aorta. Dentro de los hallazgos, se encontró que ambos grupos tenían una prevalencia de hipertensión arterial del $50 \%$ sin diferencia entre los grupos y durante el seguimiento se presentó una alta incidencia de recoartación, mayor en el grupo de angioplastia con balón. En nuestro estudio no se encontraron diferencias significativas en la incidencia de hipertensión arterial entre los grupos con o sin implantación de stent.

Otro factor relacionado con la recoartación de aorta es el gradiente final de angioplastia. Un estudio realizado en adultos con coartación nativa que recibieron manejo percutáneo mostró que la recoartación se asociaba con el gradiente residual inmediato ${ }^{23}$. Si bien objetivo final es alcanzar gradientes menores a 20 $\mathrm{mmHg}$, ya sea mediante angioplastia sola o colocando un stent, en una proporción de pacientes esto no se consigue. Tyagy, et al. reportaron que en el 7.0 al $28 \%$ de los pacientes adolescentes y adultos jóvenes sometidos a angioplastia con balón no se alcanzó un gradiente $<20 \mathrm{mmHg}^{24}$, lo cual coincide con nuestros hallazgos, los cuales indican que en el grupo sin stent fue significativamente menor la proporción de pacientes que quedaron con un gradiente $>20 \mathrm{mmHg}$. En un estudio realizado por Zabal, et al. observaron una diferencia significativa entre la angioplastia sola y el stent primario (gradiente residual 10.7 (10.8) vs. 2.7 (4.3) $\mathrm{mmHg}$, respectivamente; $p=0.001$ ) y refieren que, en pacientes con una anatomía desfavorable, la colocación de stent primario proporciona un resultado inmediato que es superior al obtenido por la angioplastia con balón solo ${ }^{23}$.

\section{Limitaciones}

Dado que la totalidad de los pacientes no fueron seguidos por un periodo de tiempo suficiente para la aparición del evento de estudio, nuestros resultados pueden subestimar la verdadera incidencia de recoartación de la aorta. El no disponer de pacientes en todas las categorías de edad con implantación de stent no permitió una comparación apropiada por esta importante variable entre los grupos comparados.

\section{Conclusiones}

La incidencia de recoartación de aorta posterior a angioplastia con balón fue menor en el grupo con implantación de stent, pero esta menor incidencia no constituye una diferencia significativa. La implantación de stent durante la angioplastia con balón para tratar coartación de aorta no influyó en el tiempo de seguimiento de esta cohorte en la recoartación de aorta; pero factores como la hipertensión arterial preangioplastia y el gradiente final de angioplastia $>20 \mathrm{mmHg}$ se asociaron con recoartación de aorta.

\section{Financiamiento}

Ninguno.

\section{Conflicto de intereses}

Ninguno.

\section{Responsabilidades éticas}

Protección de personas y animales. Los autores declaran que para esta investigación no se han realizado experimentos en seres humanos ni en animales.

Confidencialidad de los datos. Los autores declaran que han seguido los protocolos de su centro de trabajo sobre la publicación de datos de pacientes.

\section{Derecho a la privacidad y consentimiento infor-} mado. Los autores han obtenido el consentimiento informado de los pacientes y/o sujetos referidos en el artículo. Este documento obra en poder del autor de correspondencia.

\section{Bibliografía}

1. Campbell M. Natural history of coartation of the aorta. Br Heart J. 1970;32:633-40.

2. Torok RD, Campbell MJ, Fleming GA, Hill KD. Coarctation of the aorta Management from infancy to adulthood. World J Cardiol. 2015;7(11):765-75

3. Valdebenito M, Martínez A, Martínez G, Veas N, Lindefjeld D, Garay F, et al. Tratamiento endovascular de la coartación aórtica nativa y recurrente en pacientes mayores de 4 años: resultados y complicaciones. Rev Chil Cardiol. 2013;32(1):34-9.

4. Sos T, Sniderman KW, Tettek-Sos B, Strupp A, Alonso DR. Percutaneous transluminal dilatation of coarctation of thoracic aorta post mortem. Lancet. 1979;2:970-1.

5. Forbes TJ, Gowda ST. Intravascular stent therapy for coarctation of the aorta. Methodist Debakey Cardiovasc J. 2014;10(2):82-7. 
6. Reich $\mathrm{O}, \operatorname{Tax} \mathrm{P}$, Bartáková $\mathrm{H}$. Long-term (up to 20 years) results of percutaneous balloon angioplasty of recurrent aortic coarctation without use of stents. Eur Heart J. 2008;29(16):2042-8.

7. Hijazi ZM, Geggel RL. Balloon angioplasty for postoperative recurrent coarctation of the aorta. J Intervent Cardiol. 1995;8:509-16.

8. Yetman AT, Nykanen D, McCrindle BW, Sunnegardh J, Adatia I, Freedom RM, et al. Balloon angioplasty of recurrent coarctation: a 12 years review. J Am Coll Cardiol. 1997:30:811-16.

9. Zabal C, Attie F, Martinez-Rios MA, Buendia A. Balloon angioplasty for native aortic coarctation: short and intermediate term results evaluated with angiography and magnetic resonance imaging. J Cardiovasc Diagn proc. 1996;13:223-32

10. Rao PS. Long-term follow-up results after balloon dilatation of pulmonic stenosis, aortic stenosis, and coarctation of the aorta: a review. Prog Cardiovasc Dis. 1999:42:59-74

11. Parra-Bravo JR, Reséndiz-Balderas M, Francisco-Candelario R. Angioplastia con balón de la coartación aórtica nativa en niños menores de 12 meses: resultado inicial y a mediano plazo. Arch Cardiol Méx. 2007;77(3):217-25.

12. Valdebenito M, Martínez A, Martínez G, Veas N, Lindefjeld D, Garay F et al. Tratamiento endovascular de la coartación aórtica nativa y recurrente en pacientes mayores de cuatro años: resultados y complicaciones. Rev Chil Cardiol. 2013;32:34-9.

13. Eraso-Díaz del Castillo, Escobar-Díaz MC, Lince Varela, Díaz Medina. Catheterization performed in the early postoperative period after congenital heart surgery in children. Pediatric Cardiology. 2019;40:827-33.

14. Vitiello R, McCrindle BW, Nykanen D, Freedom RM, Benson LN. Com plications associated with pediatric cardiac catheterization. J Am Col Cardiol. 1998;32(5):1433-40.
15. González Álvarez C, Ordóñez Toquero G, Rivera Quintero E. Angioplastia con stent en el tratamiento de coartaciones aórticas críticas. Arch Cardiol Méx. 2006;76(4).

16. Pedra CA, Fontes VF, Esteces CA Stenting vs balloon angioplasty for discrete unoperated coarctation of the aorta in adolescents and adults. Cathet Cardiovasc Interv. 2005;64:495-506.

17. Bermúdez-Cañete R. Coartación de aorta: posibles soluciones a un complejo problema. Rev Esp Cardiol. 2005:58(9):1010-3.

18. Bulbul ZR, Bruckheimer E, Love JC, Fahey JT, Hellenbrand WE. Implantation of balloon-expandable stents for coarctation of the aorta; implantation data and short-term results. Cathet Cardiovasc Diagn. 1996;39:36-42.

19. Zahn EM, Dobrolet NC, Nykanen DG, Ojito J, Hannan RL, Burke RP. Interventional catheterization performed in the early postoperative period after congenital heart surgery in children. J Am Coll Cardiol. 2004;43(7):1264-12.

20. Nicholson GT, Kim DW, Vincent RN, Kogon BE, Miller BE, Petit CJ. Cardiac catheterization in the early post-operative period after congenital cardiac surgery. JACC Cardiovasc Interv. 2014;7(12):1437-43.

21. Dijkema EJ, Sieswerda GJT, Takken T, Leiner T, Schoof PH, Haas F, et al. Long-term results of balloon angioplasty for native coarctation of the aorta in childhood in comparison with surgery. Eur $\mathrm{J}$ Cardiothorac Surg. 2018;53:262-8.

22. Swartz MF, Morrow D, Tallah-Yunes N, Cholette JM, Gensini F, Kavey RE, et al. Hypertensive changes within the aortic arch of infants and children with isolated coarctation. Ann Thorac Surg. 2013;96:190-5.

23. Zabal C, Attie $F$, Rosas M, Buendía-Hernández A, García-Montes JA The adult patient with native coarctation of the aorta: balloon angioplasty or primary stenting? 2002. Heart. 2003;89:77-83.

24. Tyagi S, Arora R, Kaul UA. Balloon angioplasty of native coarctation of the aorta in adolescents and young adults. Am Heart J. 1992;123:674-80. 